\title{
Éditorial
}

\section{Dosage et spéciation des métaux dans les milieux biologiques d'hier à aujourd'hui}

\section{Determination and speciation of metals in biological media : from yesterday to nowa days}

Pierre ALLAIN

Laboratoire de Pharmacologie et Toxicologie, CHU - 49033 ANGERS Cedex 01

(Reçu le 10 mai 2001 ; accepté le 20 juin 2001)

e me propose de faire le point sur l'évolution des méthodes de dosage et de spé-
ciation des métaux dans les milieux biologiques d'hier à aujourd'hui, à travers
mon expérience personnelle.

Lorsque j'ai pris la responsabilité du laboratoire de pharmacologie au CHU d'Angers en 1970, les techniques utilisées pour le dosage des éléments (métaux) reposaient essentiellement sur les réactions colorées. Le dosage du sodium et du potassium se faisait cependant déjà en spectrométrie d'émission de flamme. La spectrométrie d'absorption atomique en flamme existait aussi, mais était peu répandue et insuffisamment sensible pour le dosage de la plupart des éléments. C'est le remplacement de la flamme par le four graphite et l'introduction de la correction d'absorption non spécifique qui a donné à la spectrométrie d'absorption atomique une grande sensibilité et lui a permis de devenir, lentement mais sûrement, une méthode de référence. Nous avons eu le premier four graphite commercialisé, le HGA70 Perkin-Elmer. Un certain nombre de difficultés, aujourd'hui bien connues, dans l'utilisation de l'absorption atomique sans flamme nous ont conduit à rechercher des techniques complémentaires. 
En 1978, nous avons commencé à utiliser l'ICP-OES (Inductively Coupled PlasmaOptical Emission Spectrometry), au moyen d'une torche plasma (Plasmatherm) couplée à un monochromateur et détecteur Jobin-Yvon. Ces montages qui nous ont permis de doser l'aluminium d'une façon fiable ont été progressivement remplacés par des ensembles plus évolués et automatisés comme le JY48. Les limites de l'ICP-OES sont apparues très rapidement : on savait qu'on ne pouvait pas -sans acrobaties parfois suggérées par les constructeurs- mesurer correctement dans les milieux biologiques les éléments comme le plomb, le cadmium, l'étain, l'antimoine. Les insuffisances de l'ICP-OES -la polarographie ne nous ayant pas donné de résultats très satisfaisants- nous ont conduit à rechercher des techniques complémentaires et nous nous sommes tournés à partir de 1985 vers l'ICP-MS (Inductively Coupled PlasmaMass Spectrometry). L'ICP-MS, à l'aide d'un prototype qui n'a pas eu de descendance, a permis de concrétiser les promesses de cette méthode. Aujourd'hui, l'ICPMS au moyen d'un ELAN 5000 Perkin-Elmer et d'un AGILENT 7500 est la méthode principale du laboratoire.

Alors le choix entre ICP-OES et ICP-MS ? L'ICP-OES reste la technique la plus adaptée pour doser des éléments présents à concentrations relativement élevées : les alcalins, les alcalino-terreux, le fer, le zinc, le cuivre et le dosage de certains éléments comme le silicium et peut-être l'aluminium, l'ICP-MS couvrant le reste des éléments.

Quant à la spéciation, elle bénéficie de la facilité du couplage de l'ICP-MS aux techniques séparatives, chromatographie liquide et électrophorèse capillaire. Ce couplage ne pose aujourd' hui aucune difficulté particulière. La difficulté provient essentiellement de la réactivité et de l'instabilité des espèces à séparer, notamment les fixations aux colonnes de séparation. La LC-ICP-MS peut dans certains cas être utilement complétée par la LC-MS et pour des produits légers volatils par la GC-MS .

En conclusion, nous avons aujourd'hui à notre disposition des méthodes permettant de mesurer très correctement la concentration de la quasi-totalité des éléments de la classification de Mendeleiev. Les progrès très rapides de la spectrométrie de masse, la banalisation des systèmes à temps de vol (TOF, time of flight) par exemple, et de l'informatique -même si les logiciels sont rarement bien conçus- nous permettent aujourd'hui de disposer d'outils extrêmement performants et automatisés. Cependant la possession de ces outils ne dispense pas de l'acquisition de connaissances fondamentales et de l'habitude des matrices dans lesquelles sont faits les dosages, si l'on veut éviter des erreurs parfois grossières. Aujourd'hui, in vitro tout est possible mais les explorations in vivo restent limitées. Est-il temps de parier sur elles ? 